\title{
Development of Anthropometric Data Base from Scanned Bodies for Improving Pattern Block
}

\author{
Victor E. KUZMICHEV ${ }^{\mathrm{a}}$, E. KOZLOVA ${ }^{* a}$ Jean-Loup RENNESSON ${ }^{\mathrm{b}}$ \\ ${ }^{a}$ Ivanovo State Textile Academy, Department of Garment Design, Ivanovo, Russia \\ ${ }^{\mathrm{b}}$ TELMAT Industrie SA, Soultz, France
}

\begin{abstract}
Modern graph-analytic methods of the pattern block making are based on the limited number of body sizes varied from 5 to 15 ones. Such small number of body sizes is enough for the basic block lines drawing and calculating some lines length belonging to the typical classification of bodies. If we use these methods for the full range of the body types with all possible sizes and heights, we will meet the difficulty in getting the balanced and good fitting clothes. The features of real bodies require the correction of the equations and the new manipulations adding for the block shaping. By means of the bodyscanning technology we can get new body sizes to improve the block patterns. We created the technology how to adopt the block pattern made by any method to the real body morphology.
\end{abstract}

Key words: bodyscanner, body sizes, pattern block, anthropometric net, clothes, fit, balance

\section{Introduction}

Existing methods of pattern block making (front, back, sleeve, etc.) are based on a limited set of body sizes. Their number that used in the analytical and graph-analytic methods of pattern drawing are changed from 5 to 15 .

Such number of body sizes is sufficient to determine the dimensions of the basic block and pattern construction but do not guarantee the adequacy to the body features. When using these techniques for a full range of standard (typical) bodies in the full range of the sizes and heights, we encounter difficulties in getting balanced clothes. In accordance with our research, only $40 \%$ block patterns may be identified as good, but $60 \%$ patterns have had the problems in the balances, fitting, and accuracy [1]. Features of individual body morphologies usually require the adjustment of the equations and additional drawing techniques.

However, the limitations of existing methods of pattern block making in getting a balanced and harmonious clothes are evident when the designers are trying to use them to the whole scale of sizes and heights. Therefore, the improvement of methods for pattern making for standard and nonstandard bodies shapes is an important task.

The most perspective way of improving the pattern block quality is the new data base which will get by the body scanners [2]. But the utilization of full information involves the serious science problem $(3 \mathrm{D} \rightarrow 2 \mathrm{D} \rightarrow 3 \mathrm{D})$ that has next aspects:

- to establish the rational number of body sizes which will allow predict all body features in the block pattern,

- to create the ways of pattern block lines drawing. We can draw the additional lines and surfaces across the scanned images which can help to adopt new body sizes more exactly. For example, the vertical surface across the shoulder point SP can help to divide all girths - bust, waist, hip - between the front and back. Vertical surfaces across the buttock point and some points belonging to waist line will establish the new body sizes which could directly used in trousers pattern block; - to choose the methods of finding the anthropometric points which must be move correctly from 3D body to 2D flat pattern.

Main goal of our research is to develop the pattern blocks which should be adequate to the real scanned body morphology.

\section{Methods}

We have considered the different ways of the calculations and combinations of additional points and lines generated from the scanned body. For drawing and checking of the pattern block we created the anthropometric net which includes the horizontal, vertical, and sloping lines in accordance with the human body morphology (fig.1).

*wkd37@list.ru; +7 9206722067 

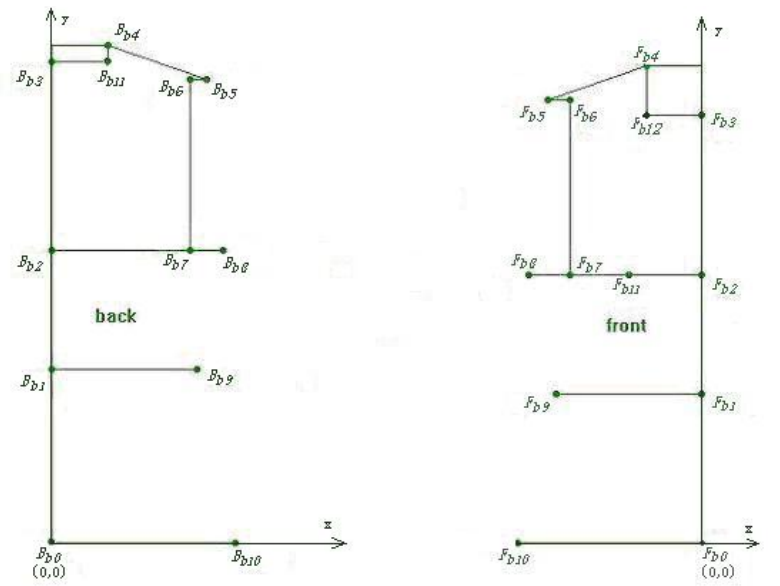

Fig. 1. Anthropometric net generated from the back and front of scanned body.

Anthropometric net is "a passport" of individual human body, the base of adequate pattern block, and the tool for checking other patterns created before. We established the new data bases for the main kinds of clothes and prepared some ways for increasing the pattern quality.

\section{Results}

To show the possibilities of anthropometric net we put together 3 different woman jacket block patterns used in Russia, Great Britain, and France. Fig.2 shown some places and number of body sizes measured from scanned woman body.

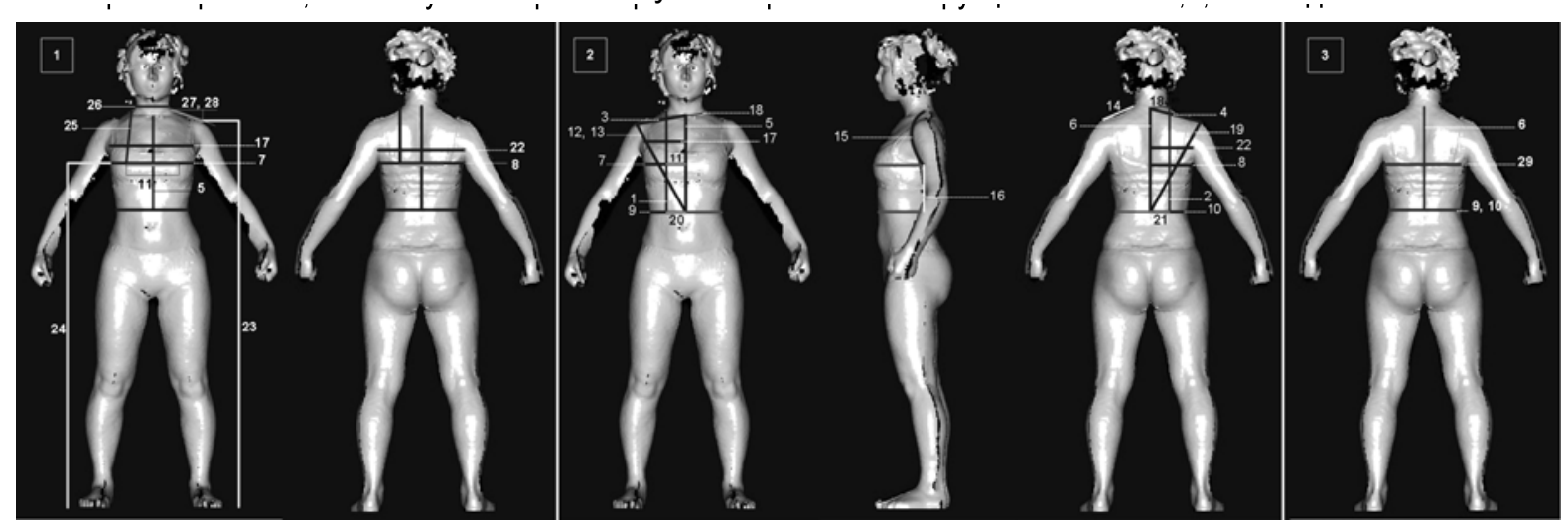

Fig.2. Anthropometric programs of body measuring used in research.

Fig.3 shown all patterns obtaining in accordance with the combination of body sizes measured (fig.2) and joining in anthropometric point SP.

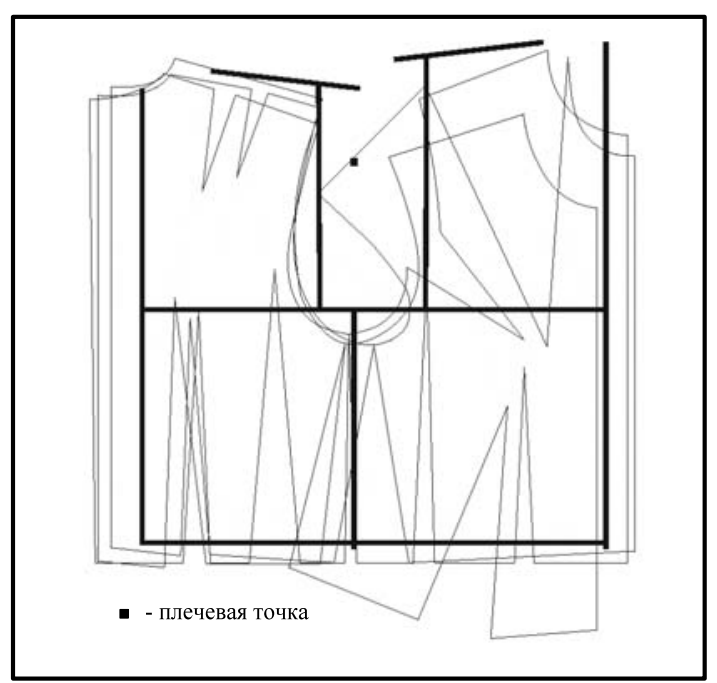

Fig. 3. Way of the patterns analyzing obtained by different methods by means of anthropometric net. 
Analysis shown the meaningful differences in pattern blocks made for the same body but by different methods. The blocks had different eases to bust girth, armhole depths, back width, bust width and angle of shoulder slope. Anthropometric net drawing in strong black line shown which pattern is more closely to the body morphology. This example had shown the weak sides of the existing methods of pattern making which are not perfect and could be improved.

To compare the different combinations of traditional and new body sizes taken from scanned body surface we calculated the indexes for the pattern blocks, such as:

- the number of points laying on the counter lines and inside the block pattern calculated by means of one, two, or three body sizes have taken from body sizes standard and new ones, - the number of triangular inside the block pattern which gain the points on the different anthropometric levels. Triangulation is the most accurate and logical graphical way to find the coordinates of anthropometric points on the plane. The principle of triangulation allows determining the exact positions of anthropometric points laying on the pattern;

- the number of operation to correct the pattern block, etc.

Fig. 4 shown the example how to use this method for coordinating some points such as (1) shoulder point SP, neck points BNP and SNP, (2) upper point of back line, and two points on the bottom line, (3) BP and some points located on the middle and bottom lines.

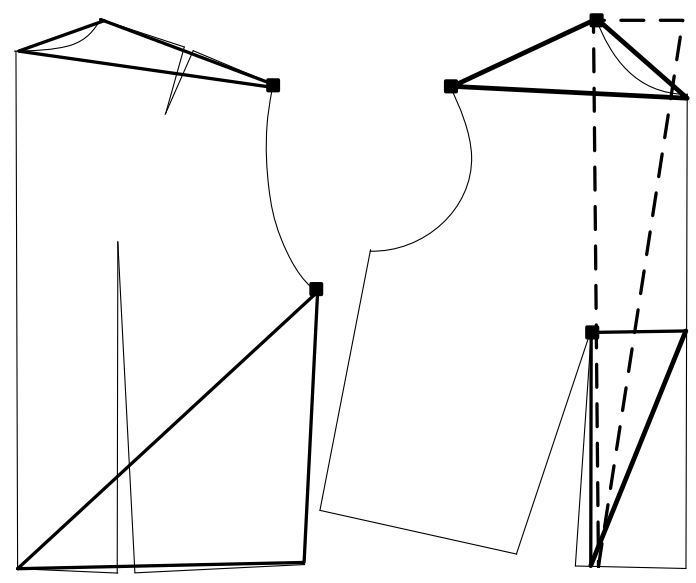

Fig. 4. Principle of triangulation using to coordinate some anthropometric points by means of additional bodies sizes measured from scanned body between new anthropometric points.

We checked at the various calculation methods and combinations of additional points and lines obtained from scanned body.

After comparative analysis of pattern bocks, some optional anthropometric programs were developed. The adequacy of pattern depends from the number of body sizes, its combination, and the accuracy of the measurements. The opportunity of targeted improvements in the adequacy of patterns opened the scanned body shapes by means of increasing the number of body sizes measured on, and measurements used in pattern drawing.

\section{Conclusion}

During our research the algorithms and adequacy of the more popular pattern blocks constructed by different methods were analyzed using the new way of body shape and morphology presentation by anthropometric net generated after bodyscanning.

We have created a new database for the main types of clothes (trousers, dresses, jackets) and prepared a number of ways to improve the clothes fit and balance in according with the scanned body morphology.

\section{References}

1. Surikova, O., Surikova G., Kuzmichev, V. (2008). Pattern blocks obtained by different methods and human bodies morphology adequacy. Швейная промышленность, № 5 (rus.)

2. Kuzmichev, V. (2012). Body scanners and clothes: new technologies in clothes design

(Бодисканеры и одежда: Новые технологии проектирования одежды). LAP LAMBERT Academic

Publishing, 2012. ISBN 978-3-659-10740-5. 